\title{
The Effect of Natural Killer Cell Killer Ig-Like Receptor Alloreactivity on the Outcome of Bone Marrow Stem Cell Transplantation for Severe Combined Immunodeficiency (SCID)
}

M. D. Keller • D. F. Chen • S. A. Condron • N. Liu •

N. L. Reinsmoen • R. H. Buckley

Published online: 22 September 2007

(C) Springer Science + Business Media, LLC 2007

The second author's name should be spelled D. F. Chen.

Throughout the text " $\mathrm{R}$ " " should be "RR" when referring to relative risk.

The online version of the original article can be found at http://dx.doi. org/10.1007/s10875-006-9058-7

M. D. Keller • R. H. Buckley

Division of Pediatric Allergy and Immunology,

Duke University Medical Center,

Durham, NC, USA

D. F. Chen $\cdot$ N. Liu $\cdot$ N. L. Reinsmoen

Clinical Transplantation Immunology Laboratory,

Duke University Medical Center,

Durham, NC, USA

\section{S. A. Condron}

Duke School of Medicine, Duke University Medical Center,

Durham, NC, USA

R. H. Buckley $(\bowtie)$

Duke University Medical Center,

P.O. Box 2898, Durham, NC, USA

e-mail: buck1003@mc.duke.edu 